\title{
Entangled Photon Pairs from Semiconductor Quantum Dots.
}

\author{
N. Akopian, N. H. Lindner, E. Poem, Y. Berlatzky, J. Avron, and D. Gershont \\ Department of Physics, Technion-Israel Institute of Technology, 32000 Haifa, Israel \\ B. D. Gerardot and P. M. Petroff \\ Materials Department, University of California Santa Barbara, CA, 93106, USA
}

\begin{abstract}
Tomographic analysis demonstrates that the polarization state of pairs of photons emitted from a biexciton decay cascade becomes entangled when spectral filtering is applied. The measured density matrix of the photon pair satisfies the Peres criterion for entanglement by more than 3 standard deviations of the experimental uncertainty and violates Bell's inequality. We show that the spectral filtering erases the "which path" information contained in the photons color and that the remanent information in the quantum dot degrees of freedom is negligible.
\end{abstract}

Entanglement, the intriguing correlations of quantum systems [1, 2, 3] is an essential resource of quantum information and communication [4, 5, 6]. Entangled photons are particularly attractive for applications due to their non interacting nature and the ease by which they can be manipulated. Polarization entangled photons are routinely produced by nonlinear optical effects $7,8,9]$, predominantly by parametric down conversion [7, 8]. Such sources have a large random component whereas quantum data processing schemes require non-random, or "event ready", entangled photons.

Semiconductor quantum dots (SCQDs) 9, 10, 11, 12, 13, 14 provide optically 14, 15] and electrically 16] driven sources of single photons "on demand". Compatibility with modern electronics makes them potential building blocks for quantum information processing in general 17 and sources for "event-ready" entangled photons 18, 19] in particular.

A SCQD biexciton decays radiatively through two intermediate optically active exciton states [20, 21]. The proposal that the biexciton-radiative cascade could provide a source of event-ready polarization entangled photon pairs was made by Benson et. al. 18]. Entanglement requires two decay paths with different polarizations, but otherwise indistinguishable. This is the case if the intermediate exciton states are energetically degenerate and if, in addition, the final state of the SCQD is independent of the decay path.

The first requirement is difficult to fulfil since the intermediate exciton states are normally split (by the electronhole exchange interaction) 22, 23. The two decay paths, which we denote by horizontal $(\mathrm{H})$ and vertical $(\mathrm{V})$, have corresponding photon polarizations relative to the asymmetry axis of the QD (shown in Fig. (1). The paths are then spectrally distinguishable and the polarization state of the photons cannot be entangled [24]. The second requirement, that the SCQD final state does not depend on the decay path, has never been tested experimentally.

In this Letter, we show for the first time [27] that the "which path" information can be erased by filtering the photons spectrally and that this procedure produces an entangled polarization state. This also proves that the remnant "which path" information residing in the myriad degrees of freedom of the SCQD, must be small.

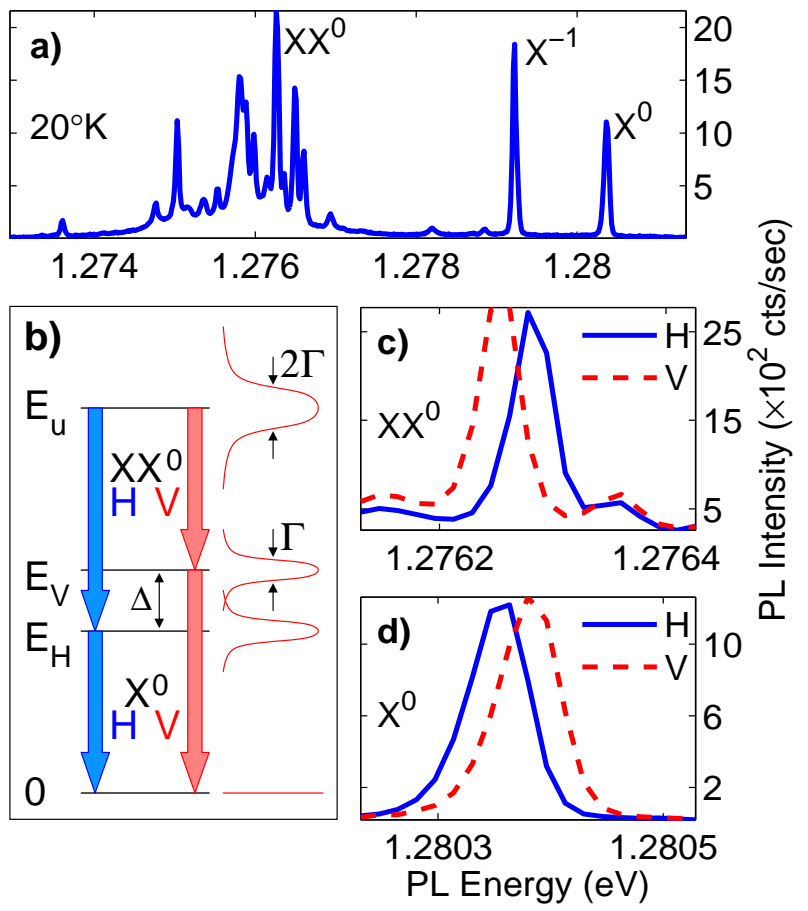

FIG. 1: a) PL spectrum of a single SCQD embedded in planar MC. (b) Schematic description of the biexciton radiative cascade in natural SCQDs with its two co-linearly polarized photons (either $\mathrm{H}$ or $\mathrm{V}$ ), with energetically distinguishable paths. (c), ((d)) High resolution polarization sensitive PL spectra of the biexciton $\mathrm{XX}^{0}$ (exciton $\mathrm{X}^{0}$ ) lines. The measured spectral width is much larger than the measured radiative width shown schematically in (b).

We denote by $|H H\rangle(|V V\rangle)$ the two photons' polarization state associated with the $\mathrm{H}(\mathrm{V})$ decay path. The final state of the system is given by

$$
|\psi\rangle=\alpha\left|p_{H}\right\rangle|H H\rangle\left|d_{H}\right\rangle+\beta\left|p_{V}\right\rangle|V V\rangle\left|d_{V}\right\rangle .
$$

Here $\alpha$ and $\beta$ are the amplitudes of the decay paths $\left(|\alpha|^{2}+|\beta|^{2}=1\right)$. The photons' wave packet is denoted 
$\left|p_{H(V)}\right\rangle$ and the final states of the SCQD by $\left|d_{H(V)}\right\rangle$. All may, a-priori, depend on the decay path.

In Eq.(11) we neglected the broken paths, $|H V\rangle$ and $|V H\rangle$, which have negligible amplitudes [28].

The density matrix for the two photons polarization state is given by tracing out the $|p\rangle$ and $|d\rangle$ degrees of freedom:

$$
\rho=\left(\begin{array}{cccc}
|\alpha|^{2} & 0 & 0 & \gamma \\
0 & 0 & 0 & 0 \\
0 & 0 & 0 & 0 \\
\gamma^{\star} & 0 & 0 & |\beta|^{2}
\end{array}\right), \gamma=\alpha \beta^{\star}\left\langle p_{H} \mid p_{V}\right\rangle\left\langle d_{H} \mid d_{V}\right\rangle .
$$

The phase of $\gamma$ is a gauge dependent quantity.

The Peres criterion [25] applied to Eq.(2) says that the polarization is entangled provided $\gamma \neq 0$. This may be reformulated in terms of the reliability of the "which path" indicator: The indicator is reliable if its interrogation selects the path, $\mathrm{H}$ or $\mathrm{V}$, with no error. This is the case if $\left|p_{H}\right\rangle\left|d_{H}\right\rangle$ is orthogonal to $\left|p_{V}\right\rangle\left|d_{V}\right\rangle$. In this case $\gamma=0$ and the state is correlated (there are no HV events) but not entangled. If $\gamma \neq 0$, interrogating the indicator does not select a unique path and the state is entangled. When $\left|p_{H}\right\rangle\left|d_{H}\right\rangle$ is parallel to $\left|p_{V}\right\rangle\left|d_{V}\right\rangle$, maximal entanglement, with $|\gamma|=\frac{1}{2}$, is obtained if the two paths have equal weights $|\alpha|^{2}=|\beta|^{2}=\frac{1}{2}$.

Entanglement need not imply violation of Bell's inequality. Horodecki et al. 29] show how to choose the Bell operator $B$ for a given $\rho$ in order to maximally violate the (CHSH) Bell inequality. Applying their result to $\rho$ yields

$$
\operatorname{Tr}(B \rho)=2 \sqrt{1+4|\gamma|^{2}} .
$$

Bell inequality is then violated for all $\gamma \neq 0$. Maximal violation of $2 \sqrt{2}$ is obtained for a maximally entangled state with $|\gamma|=\frac{1}{2}$.

Suppose $\left\langle p_{H} \mid p_{V}\right\rangle=0$. Then $\gamma=0$ and the state is not entangled. Nevertheless, one can entangle such a state by applying a projection $P$ on the wave packet. This replaces $|\psi\rangle$ of Eq. (11) by $P|\psi\rangle /|P| \psi\rangle \mid$ and gives

$$
\gamma^{\prime}=\frac{\alpha \beta^{\star}\left\langle p_{H}|P| p_{V}\right\rangle}{|P| \psi\rangle\left.\right|^{2}}\left\langle d_{H} \mid d_{V}\right\rangle .
$$

An optimal choice is such that $\alpha^{\star} P\left|p_{H}\right\rangle=\beta^{\star} P\left|p_{V}\right\rangle$ giving in turn $\gamma^{\prime}=\frac{1}{2}\left\langle d_{H} \mid d_{V}\right\rangle$. Maximal entanglement is obtained when the final state of the dot $|d\rangle$ does not depend on the decay path. Thus, $\left|\gamma^{\prime}\right|$ can be significant, even if $\gamma$ is negligibly small. If the final state of the SCQD can reliably distinguish between the two possible decay paths (for example, due to involvement of different phonons or spins in the radiative cascade) no entanglement will arise.

Calculating $|p\rangle$ in second-order perturbation theory within the dipole and rotating wave approximation [30] gives

$$
A_{H} \equiv \alpha\left\langle\mathbf{k}_{1}, \mathbf{k}_{2} \mid p_{H}\right\rangle=\frac{e^{i \phi_{H}} \Gamma / 2 \pi}{\left(\left|\mathbf{k}_{1}\right|+\left|\mathbf{k}_{2}\right|-\epsilon_{u}\right)\left(\left|\mathbf{k}_{2}\right|-\epsilon_{H}\right)},(5)
$$

with a similar expression for $A_{V}$. The index $u$ denotes the initial biexciton state. The momentum of the photons is labelled $\mathbf{k}_{1}, \mathbf{k}_{2}$ (using units in which $\hbar=c=1$ ), and $\epsilon_{j}=E_{j}-\frac{i}{2} \Gamma_{j}, j=u, H, V$ are complex energies with radiative widths $\Gamma_{j}$. We implement $P$ as a projection of the second photon on a rectangular window function $W\left(\mathbf{k}_{2}\right)$ of width $w$, centered at the average intermediate energy $\left(E_{V}+E_{H}\right) / 2$. Plugging this into Eqs. (4) gives

$$
\gamma^{\prime}=\frac{\iint d \mathbf{k}_{1} d \mathbf{k}_{2} A_{H}^{\star} W A_{V}}{\iint d \mathbf{k}_{1} d \mathbf{k}_{2} A_{H}^{\star} W A_{H}+\iint d \mathbf{k}_{1} d \mathbf{k}_{2} A_{V}^{\star} W A_{V}} .
$$

The denominator, $|P| \psi\rangle\left.\right|^{2}$, is the detection probability of the two-photons within the spectral window $W$.

From Eq. (6) we calculate $\gamma^{\prime}$ as a function of $w$ with $\Gamma_{u}=2 \Gamma_{H / V}$ and the detuning $\Delta=E_{H}-E_{V}$ taken from our measurement. Although one can easily calculate $\gamma^{\prime}$ exactly (see Fig.4) the result is more transparent when one takes into account that the radiative width $\Gamma$ is the smallest energy scale in the problem. When the window $w$ is smaller than the detuning, $\Delta, \gamma^{\prime}$ is actually independent of the radiative width

$$
\left|\gamma^{\prime}\right|=\frac{1}{4}\left(\frac{1}{x}-x\right) \log \left(\frac{1+x}{1-x}\right), \quad x=\frac{w}{\Delta} .
$$

$\left|\gamma^{\prime}\right|$ reaches the maximal value of $\frac{1}{2}$ when the window is vanishingly small. However, the detection probability $|P| \psi\rangle\left.\right|^{2}$ vanishes in this limit as well. In the opposite limit of no filtering, when the window is large, the detection probability $|P| \psi\rangle\left.\right|^{2}$ approaches unity, while $\left|\gamma^{\prime}\right|$ approaches the residual (small) value $\Gamma / 2 \Delta$. The calculation applies a single spectral window for the second photon (energy conservation is then sufficient to spectrally project the first photon).

For the measurements we used planar microcavity (MC) embedded SCQDs samples. The samples were grown by molecular beam epitaxy on a (100) oriented GaAs substrate. One layer of strain-induced InAs QDs was deposited in the center of a one wavelength GaAs microcavity formed by two unequal stacks of alternating quarter wavelength layers of AlAs and GaAs, respectively. The height and composition of the QDs were controlled by partially covering the InAs QDs with a $3 \mathrm{~nm}$ layer of GaAs and subsequent growth interruption. To improve photon collection efficiency, the microcavity was designed to have a cavity mode, which matches the QD emission due to ground state e-h pair recombinations. The sample was not patterned laterally to prevent obscuration of the emitted photon polarizations.

We used a diffraction limited low temperature confocal optical microscope for the photoluminescence (PL) studies of single MCQDs 11, 21]. Temporal correlations between emitted photon pairs were measured using a wavelength and polarization selective Hanbury-Brown-Twiss 
(HBT) arrangement 21]. We used a 1 meter monochromator in each arm of the HBT setup to obtain spectral resolution of $\sim 15 \mu \mathrm{eV}$. The polarization state of the emitted light was monitored by the use of liquid crystal variable retarders (LCVRs) and high quality polarizers.

In Fig. 1h we present PL spectrum of a single, resonant MCQD. The MCQD was excited by a continuouswave HeNe laser. The spectrum is composed of sharp lines with linewidths of roughly $50 \mu e v$ due to inhomogeneous broadening caused by temporal changes in the SCQD electrostatic environment. We identified most of the observed spectral lines using power and energy dependence polarization sensitive magneto spectroscopy. Here, we are only interested in the neutral single exciton line $\left(\mathrm{X}^{0}\right)$ and the neutral biexciton line $\left(\mathrm{XX}^{0}\right)$. In Fig $1 \mathrm{c}$ and $1 d$ we present high resolution polarization sensitive PL spectra of the lines $\mathrm{X}^{0}$ and $\mathrm{XX}^{0}$. Fig. 1] demonstrates that the neutral spectral lines $\mathrm{XX}^{0}$ and $\mathrm{X}^{0}$ are composed of two cross linearly polarized split doublets with detuning of $\Delta=27 \pm 3 \mu \mathrm{eV}$. We used the HBT setup to

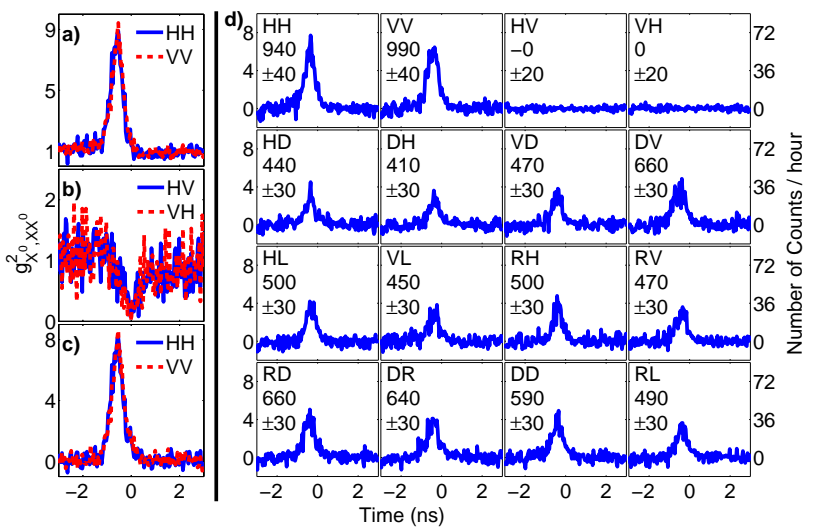

FIG. 2: a) (b)) The temporal intensity cross correlation measurements between the exciton $\mathrm{X}^{0}$ and the biexciton $\mathrm{XX}^{0}$ spectral lines for co- (cross-)linear polarizations. c) The reduced intensity correlation measurements obtained by the difference between the collinearly polarized curves in a) and the average curve of the cross-linearly polarized measurements in b). d) Tomographical measurements of the 'reduced' intensity cross-correlation functions with spectral resolution of $25 \mu \mathrm{eV}$. $\mathrm{D}$ stands for linear polarizer at $45^{\circ}$ relative to the $\mathrm{H}$ direction and $\mathrm{R}(\mathrm{L})$ stands for right (left) hand circular polarizer. The integrated numbers of coincidences in each measurement are indicated.

measure polarization sensitive temporal intensity correlations between photons emitted from all the observed spectral lines. The auto-correlation measurements of the lines (not shown), show a deep anti-bunching notch at coinciding times $(\mathrm{t}=0)$, demonstrating that each line is a spectral source of single photons $14,15,16]$. The intensity cross correlation measurements between the neutral exciton $\mathrm{X}^{0}$ and the neutral biexciton $\mathrm{XX}^{0}$ lines are presented in Fig. 22 (2)) for two different co- (cross-) linear polarization arrangements. For these measurements, the excitation intensity was tuned such that both lines were essentially equal in strength. When the two polarizers are co-linearly oriented along the major QD axes (HH or $\mathrm{VV})$ an asymmetric trace is obtained in which the positive temporal part shows an anti-bunching notch, while the negative part shows a strong, enhanced bunching peak. This asymmetrical shape, an experimental signature of an optical cascade, reveals the temporal sequence of these events. While emission of a horizontally (vertically) polarized $\mathrm{XX}^{0}$ photon is followed by emission of a horizontally (vertically) polarized $\mathrm{X}^{0}$ photon, the opposite never happens [18, 19]. When the polarizers are cross linearly polarized the bunching trace at negative times, is replaced by an anti-bunching-like trace. This is exactly as anticipated by the considerations of Fig. 11. The linear polarization states of the two photons emitted during the biexciton-exciton recombination cascade are completely correlated and both are collinearly polarized.

From the temporal correlation measurement we obtain the radiative lifetime of the exciton 21] $T_{X^{0}}=0.8 \pm$ $0.2 \mathrm{nsec}$, which yields $\Gamma=1.6 \pm 0.4 \mu \mathrm{eV}$ for the excitonic radiative width.

In Fig. 21) we present the "reduced" intensity correlation functions, obtained by subtracting the average of the cross-linearly polarized traces from the traces of all the tomographic correlation measurements. By integrating the "reduced" functions over time we obtain the net number of cascaded coincidences, used for the tomography.
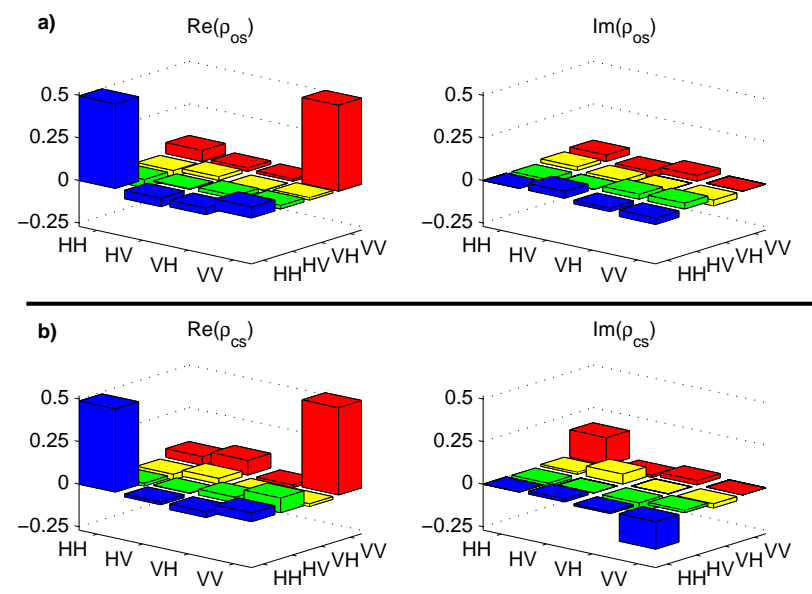

FIG. 3: The measured two photons' density matrix: a) (b)) obtained with spectral window of $200(25) \mu \mathrm{eV}$.

We performed two sets of independent measurements of the reduced intensity cross correlation function in 16 different combinations of the polarizers in front of the two detectors. The spectral projections of the photons was implemented by both monochromators' slits to secure against the inhomogeneous broadening, by effectively closing the window as it meanders. In the first 
set the monochromators' slits were opened to spectral resolution of $200 \mu \mathrm{eV}$, as independently determined by measuring the spectral lines of a low pressure mercury lamp. In the second set the resolution was set to $25 \mu \mathrm{eV}$. This set is shown in Fig. 2 $\mathrm{d}$ ). The normalized integrated numbers of coincidences from these polarization quantum tomography measurements [33] are then used to generate the density matrices in the $\mathrm{H} \mathrm{V}$ basis, as displayed in Fig. 3 $\mathrm{k}$ for the first set and Fig. 3b for the second. The experimentally obtained density matrices was then fitted to the form of Eq. (2). In the first case we obtained $|\alpha|^{2}=|\beta|^{2}=0.50 \pm 0.02$ and $\gamma^{\prime}=0.03 \pm 0.04+i(0.00 \pm 0.04)$. This agrees with the theoretical estimate of $\left|\gamma^{\prime}\right|=\Gamma / 2 \Delta \approx 0.03$. In the second case we obtained $|\alpha|^{2}=|\beta|^{2}=0.50 \pm 0.04$ and $\gamma^{\prime}=0.05 \pm 0.05+i(0.17 \pm 0.05)$ hence $\left|\gamma^{\prime}\right|=0.18 \pm 0.05$ is significantly different from zero. The photon pairs are therefore entangled with confidence level greater than 3 standard deviations of the measurement uncertainty. Substituting $\left|\gamma^{\prime}\right|$ in Eq. (3) gives $2.13 \pm 0.07$ which violates Bell's inequality.

In Fig. 4 we plot the calculated values of the normalized projected coincidences $|P| \psi\rangle\left.\right|^{2}$ and $\left|\gamma^{\prime}\right|$ as a function of the spectral window width $w$. The measured data points at $25 \mu \mathrm{eV}$ and $200 \mu \mathrm{eV}$ agree to within the error bars with the calculations. This confirms that $\left|\left\langle d_{H} \mid d_{V}\right\rangle\right| \approx 1$. Thereby, we have verified that no "which path" information is contained in the final state of the SCQD.

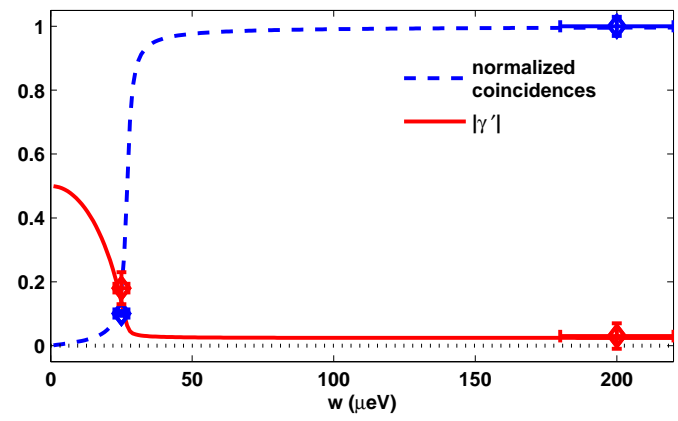

FIG. 4: Measured (symbols) and calculated (lines) normalized number of coincidences $|P| \psi\rangle\left.\right|^{2}$ and off diagonal density matrix element $\left|\gamma^{\prime}\right|$ vs. the spectral window width . For the calculations we used $\Gamma=1.6 \mu \mathrm{ev}$ and $\Delta=27 \mu \mathrm{eV}$ as experimentally determined (see text).

The entangled photons generated in this work are not event ready for two reasons: First, we used continuous excitation and second, the erasure introduces randomness. However, neither is fundamental. The excitation can be triggered on demand and the randomness can be overcome by spectrally monitoring the discarded photons without demolishing the entangled pair. Reducing the detuning and increasing the radiative width is yet another approach. Indeed, QDs with significantly smaller detuning than ours have been reported [23] and, at the same time, a substantial increase in the radiative width can be achieved in SCQD through the Purcell effect 15,30 .

In summary, we demonstrated that the biexciton - exciton radiative cascade in single semiconductor quantum dots can be used as a source for entangled photon-pairs. By applying the Peres criterion to the quantum tomographic data we prove that the polarization state is entangled. Moreover, it violates Bell's inequality. The measured degree of entanglement agrees with the calculated radiative amplitudes. This proves that the which path information residing in the photons color can be erased, and, at the same time, that there is no "which path" information left in the quantum dot.

We acknowledge support by the Israel Science and by the US-Israel Binational Science Foundations.

* Electronic address: dg@physics.technion.ac.il

[1] A. Einstein, B. Podolsky and N. Rosen, Phys. Rev. 44, 777 (1935).

[2] J. S. Bell,Rev. Mod. Phys. 40, 229 (1968).

[3] J. F. Clauser et. al., Phys. Rev. Lett. 24, 549 (1970)

[4] C. H. Bennet and G. Brassard, in IEEE Int. Conf. on Computers, Systems and Signal Processing (1984).

[5] A. Ekert, Phys. Rev. Lett. 67, 661 (1991)

[6] C. H. Bennett et. al., Phys. Rev. Lett 70, 1895 (1993).

[7] D. Bouwmeester et. al., Nature 390, 575 (1997).

[8] P. G. Kwiat et. al.,Phys. Rev. A 60, 773(R) (1999).

[9] K. Edamatsu, et. al., Nature 431, 167 (2004).

[10] L. Landin et. al., Science 280, 262 (1998)

[11] E. Dekel et. al.,Phys. Rev. Lett., 80, 4991 (1998).

[12] M. Bayer et. al., Nature 405, 923 (2000).

[13] R. J. Warburton, et. al., Nature 405, 926 (2000).

[14] P. Michler et. al., Science 290, 2282 (2000)

[15] C. Santori et. al., Nature 419, 594 (2002).

[16] Z.Yuan et al, Science 295,102 (2001).

[17] D. Loss. and D. DiVincenzo, Phys. Rev. A 57, 120 (1998).

[18] O. Benson et. al., Phys. Rev. Lett. 84, 2513 (2000).

[19] D. Fattal et. al. Phys. Rev. Lett. 92, 037903 (2004).

[20] E. Moreau et. al. Phys. Rev. Lett 87, 183601 (2001).

[21] D.V. Regelman et al, Phys. Rev. Lett. 87,257401 (2001)

[22] D. Gammon et. al., Phys. Rev. Lett. 76, 3005 (1996).

[23] M. Bayer et. al., Phys. Rev. B 65, 195315 (2002).

[24] T.M. Stace et. al., Phys. Rev. B 67, 085317 (2003).

[25] A. Peres, Phys. Rev. Lett. 77, 1413, 1996.

[26] M. Horodecki, P. Horodecki, and R. Horodecki, Phys. Lett. A 223, 1 (1996)

[27] R. M. Stevenson et. al., Nature 439 179, (2006). This paper, which appeared after the present work was put on the web (quant-ph/0512048), claims to have produced polarization entangled photons by forcing degeneracy on the exciton levels. It is easy to verify, using the Peres criterion, that their measured density matrices are not entangled.

[28] M. Kroutvar et. al., Nature 432, 81 (2004).

[29] M. Horodecki et. al., Phys. Lett. A 223,1 (1996)

[30] C. Cohen-Tannoudji, J. Dupont-Roc and G. Grynberg, Atom-Photon Interactions, John Wiley \& Sons (1992). 
[31] P. M. Visser et. al.,Phys. Rev. A, 68, 053805, 2003.

[32] K.J. Vahala, Nature 424, 839 (2003).

[33] D. F. V. James et. al., Phys. Rev. A 64, 052312 (2001). 